\title{
SUBSEQUENCES OF SEQUENCES OF RANDOM VARIABLES
}

\author{
BY DAVID J. ALDOUS ${ }^{1}$
}

Communicated by Daniel W. Stroock, September 2, 1976

Chatterji [2] has formulated the following heuristic principle: given any limit property for independent identically distributed random variables (i.i.d.r.v.'s), there exists an analogous property such that an arbitrary sequence of r.v.'s always has some subsequence possessing this analogous property. By 'arbitrary', we mean that no assumption concerning dependence is made, though it may be necessary to assume moment conditions on the r.v.'s. The purpose of this note is to announce Theorem 1, which makes this principle precise in the case where the property is an "a.s. limit theorem", a concept we formalise below.

Let $P(R)$ denote the space of probability measures on $R$. For $\Pi \in P(R)$, let

$$
\begin{aligned}
\alpha(\Pi) & =\infty & & \text { if } \int|x| \Pi(d x)=\infty, \\
& =\int x \Pi(d x) & & \text { otherwise, }
\end{aligned}
$$

and let $\beta(\Pi)=\int(x-\alpha(\Pi))^{2} \Pi(d x)$.

Define a statute to be a measurable subset $A$ of $P(R) \times R^{\infty}$ such that, whenever $\Pi \in P(R)$ and $\left\{X_{n}\right\}$ is i.i.d. with distribution $\Pi$, then ( $\Pi, X_{1}(\omega)$, $\left.X_{2}(\omega), \ldots\right) \in A$ a.s. For example, the statutes corresponding to the strong law of large numbers and the law of the iterated logarithm are

$$
\begin{aligned}
& A_{1}=\left\{(\Pi, \mathbf{x}): \alpha(\Pi)=\infty \text { or } \lim _{n \rightarrow \infty} \sum_{i=1}^{n} x_{i} / n=\alpha(\Pi)\right\} . \\
& A_{2}=\left\{(\Pi, \mathbf{x}): \beta(\Pi)=\infty \text { or } \limsup _{n \rightarrow \infty} \frac{\sum_{j=1}^{n} x_{i}-n \alpha(\Pi)}{(2 n \log \log (n))^{1 / 2}}=\beta^{1 / 2}(\Pi)\right\} .
\end{aligned}
$$

Here $\mathbf{x}=\left(x_{i}\right)$ denotes a generic point in $R^{\infty}$.

It seems clear that any "a.s. limit theorem for i.i.d.r.v.'s" may be represented by a statute. We need the following technical condition, which is satisfied by the statutes corresponding to most nontrivial such theorems.

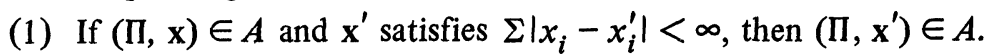

AMS (MOS) subject classifications (1970). Primary 60F15; Secondary 28A65.

${ }^{1}$ This research was supported by a grant from the Science Research Council. 
THEOREM 1. Let $\left\{X_{i}\right\}$ be a tight sequence of r.v.'s defined on a probability space $(\Omega, F, P)$. Then there exists a measurable function $\mu:(\Omega, F, P) \rightarrow P(R)$ with the following property.

Let $A$ be any statute satisfying (1). Then there exists a subsequence $\left\{X_{m_{i}}\right\}$ all of whose subsequences $\left\{X_{n_{i}}\right\}$ satisfy $\left(\mu(\omega), X_{n_{1}}(\omega), X_{n_{2}}(\omega), \ldots\right) \in A$ a.s.

To apply this theorem we need a technical fact: if $\sup _{i} E\left|X_{i}\right|^{p}<\infty$, then $\mu(\omega)$ has finite $p$ th moment a.s. As an example, let us apply Theorem 1 to the statute $A_{1}$ corresponding to the strong law of large numbers. We obtain the following result. If $\left\{X_{i}\right\}$ is a sequence of r.v.'s such that $\sup _{i} E\left|X_{i}\right|<\infty$, then there exists $\left\{X_{m_{i}}\right\}$ such that

$$
\lim _{n \rightarrow \infty} n^{-1} \sum_{i=1}^{n} X_{k_{i}}(\omega)=\alpha(\mu(\omega)) \quad \text { a.s. }
$$

for each subsequence $\left\{X_{k_{i}}\right\}$ of $\left\{X_{m_{i}}\right\}$. This gives a result of Komlós [5]. Chatterji ([2] and references therein) has proved his principle for various theorems such as the law of the iterated logarithm. All these results (except for the central limit theorem) are special cases of Theorem 1. Chatterji's technique is to reduce the case of a general sequence $\left\{X_{i}\right\}$ to the case of a martingale difference sequence, and then to use methods applicable to the particular theorem under consideration. Obviously this technique cannot prove a general result like Theorem 1, which applies to statutes representing theorems for i.i.d.r.v.'s that are as yet undiscovered!

The idea of our proof is to extract a subsequence which is "close to" an exchangeable sequence $\left\{Z_{i}\right\}$. It is essentially known that for such $\left\{Z_{i}\right\}$ there exists $\mu:(\Omega, F, P) \rightarrow P(R)$ such that for each statute $A,\left(\mu(\omega), Z_{1}(\omega), Z_{2}(\omega)\right.$, $\ldots) \in A$ a.s.

It has been suggested that the following result, which would immediately imply Theorem 1 , might be true.

CONJECTURE. Let $\left\{X_{i}\right\}$ be a tight sequence of r.v.'s. Then, by enlarging the probability space if necessary, there exists an exchangeable sequence $\left\{Z_{i}\right\}$ such that for some subsequence $\left\{X_{n_{i}}\right\}$

$$
P\left(\left|X_{n_{i}}-Z_{i}\right|>2^{-i}\right)<2^{-i} \text { for each } i \text {. }
$$

A counterexample to this conjecture will be given. A weaker result is known (Dacunha-Castelle [3]): $\left\{X_{n_{i}}\right\}$ can be chosen such that $\left(X_{n_{i+1}}, \ldots\right.$, $\left.X_{n_{i+k}}\right) \stackrel{\mathfrak{D}}{\rightarrow}\left(Z_{1}, \ldots, Z_{k}\right)$ as $i \rightarrow \infty$, for each $k$. However, this result is not strong enough to imply Theorem 1 . The proof of Theorem 1 involves choosing $\left\{X_{n_{i}}\right\}$ "close to" $\left\{Z_{i}\right\}$ in a certain, rather complicated, intermediate sense.

It is natural to look for a general result, analogous to Theorem 1, which deals with theorems about convergence in distribution. But the author can find no natural way to formulate such a result. However, the techniques used in the 
proof of Theorem 1 enable the subsequence principle for Donsker's Theorem (see Billingsley [1]) to be proved, and this is the deepest "convergence in distribution" theorem that the author knows.

These techniques also yield a proof of the following result stated by Révész [6, Theorem 5.1.1], whose proof is fallacious, as observed in [4].

THEOREM 2. Let $\left\{X_{i}\right\}$ be a sequence of r.v.'s such that $\sup _{i} E X_{i}^{2}<\infty$. Then there exists a subsequence $\left\{X_{n_{i}}\right\}$ and a r.v. $\alpha$ such that

$$
\sum_{i=1}^{\infty} a_{\sigma(i)}\left(X_{n_{\sigma(i)}}-\alpha\right)
$$

converges a.s. for each sequence $\left\{a_{i}\right\}$ of constants such that $\Sigma a_{i}^{2}<\infty$ and for each permutation $\sigma$ of the positive integers.

Proofs of these results will appear elsewhere.

\section{REFERENCES}

1. P. Billingsley, Convergence of probability measures, Wiley, New York, 1968. MR 38 \#1718.

2. S. D. Chatterji, A subsequence principle in probability theory, Bull. Amer. Math. Soc. 80 (1974), 495-497. MR 49 \#9916.

3. D. Dacunha-Castelle, Indescernability and exchangeability in $L^{p}$-spaces, Proc. Sem. Random Series, Convex Sets and Geometry of Banach Spaces, Aarhus Univ. Various Publ. 25 (1975), 50-56.

4. V. R. Gapoškin, Convergence and limit theorems for subsequences of random variables, Teor. Verojatnost. i Primenen 17 (1972), $401-423$ = Theor. Probability Appl. 17 (1972), 379-399. MR 46 \#10046.

5. J. Komlós, A generalization of a problem of Steinhaus, Acta. Math. Acad. Sci. Hungar. 18 (1967), 217-229. MR 35 \#1071. \#6391.

6. P. Révész, The laws of large numbers, Academic Press, New York, 1968. MR 39 ENGLAND

STATISTICAL LABORATORY, UNIVERSITY OF CAMBRIDGE, CAMBRIDGE, 